\title{
The Effects of Preoperative Imaging on Surgical Outcome for Pediatric Appendicitis Patients Special Issue: Pediatric Surgery
}

\author{
Henry Su ${ }^{1}$, Laura Nally ${ }^{2}$, Jennifer Maddison ${ }^{2}$, Yi-Horng Lee ${ }^{2 *}$ \\ ${ }^{1}$ Strong Children's Research Center, Department of Pediatrics, Golisano Children's Hospital, University of Rochester Medical Cen- \\ ter, Rochester, USA; ${ }^{2}$ Division of Pediatric Surgery, Department of Surgery, Golisano Children's Hospital, University of Rochester \\ Medical Center, Rochester, USA. \\ Email: *Yi-Horng_Lee@urmc.rochester.edu
}

Received May $16^{\text {th }}, 2013$; revised June $18^{\text {th }}, 2013$; accepted June $28^{\text {th }}, 2013$

Copyright (C) 2013 Henry Su, et al. This is an open access article distributed under the Creative Commons Attribution License, which permits unrestricted use, distribution, and reproduction in any medium, provided the original work is properly cited.

\begin{abstract}
Computed tomography (CT) has been the major imaging modality for the diagnosis of appendicitis because of its excellent imaging resolution and the ease of interpretation, but a concern has been raised regarding radiation exposure. We have shifted from CT to ultrasonography (US) as the primary imaging modality because of this concern. A retrospective review is conducted to determine whether a combined US/CT strategy can reduce radiation exposure while maintaining the same surgical outcome. Five hundred eighty-six patients between 10 and 18 years of age who underwent an appendectomy from 2007-2011wereincluded in our study. The patients were classified as Early (prior to 7/1/2009) or Late (after 7/1/2009) with the knowledge that a conscientious institutional effort was made to start utilizing US as the primary imaging modality, limiting CT use to selective cases in 2009. The diagnostic imaging studies performed (CT, US or no imaging) were recorded. Operative findings, pathology reports and clinical course were reviewed. The rates of acute appendicitis (AA), negative appendectomies (NA) and perforated appendicitis (PA) were calculated. During the Early period, $49.3 \%$ of patients $(n=150)$ had undergone CT scans prior to their appendectomy whereas only $37.9 \%(n$ $=107$ ) had CT scans during the Late period. Of the CT scans obtained during the Late period, only $18.4 \%$ of patients (n = 52) had a CT scan first, and 55 CT scans were done after an initial US. Patient disease patterns were similar between Early and Late years. While no difference in NA rates for boys was found between the Early and Late periods, a significantly higher incidence of NA rates was found in girls in the Early period as opposed to the Late period. We conclude that a shift in practice patterns to reduce radiation exposure for pediatric patients with appendicitis has not resulted in inferior surgical outcome.
\end{abstract}

Keywords: Appendicitis; Computed Tomography; Ultrasonography; Radiation Exposure

\section{Introduction}

Acute appendicitis is the most common pediatric surgical emergency in America [1]. The diagnosis can be challenging to make because the initial presentation of the disease can be variable or confused with other causes of abdominal pain. Furthermore, a delay in diagnosis can lead to rupture of the inflamed appendix and subsequent peritonitis. On the other hand, it is also desirable to avoid an overly aggressive approach that may result in unnecessary surgical interventions. Early reports have recorded a perforation rate of $21 \%$ and negative appendectomy

\footnotetext{
${ }^{*}$ Corresponding author.
}

rates of 20\% [2]. The necessity for making a prompt and accurate diagnosis of appendicitis has thus driven the increased utilization of radiographic imaging.

High sensitivity and specificity values for the diagnosis of appendicitis in children has been reported in the medical literature for computed tomography (CT) and ultrasonography (US), making them common adjunct tools to clinical exam [3]. The use of CT scan for the diagnosis of appendicitis has evolved from being an avantgarde diagnostic tool [4-6] to a common practice [7]. On the other hand, utilization of US for this disease started in the early 1980's [8-10], but its widespread use has been hampered by the lower diagnostic accuracy $[11,12]$ and less confidence from radiologists [13]. 
Greater awareness of CT-related cancer risks for children has made radiation exposure a cause for concern about over utilization [14,15]. As a result, a staged approach using US as the primary imaging modality has been utilized to reduce radiation exposure to children, and the experiences have previously been described [16, 17]. However, these studies do not demonstrate the institutional learning curve during adaptation to the change in practice, nor do they provide an outcome comparison between the approaches.

Our institution made a conscientious effort to reduce radiation and made a practice change in the evaluation of children presenting with abdominal pain in 2009. Instead of using the CT scan as the primary imaging modality, it is now reserved for cases with equivocal findings on either clinical exam or US. Our study is a retrospective review of patient outcomes before and after the changes took place.

\section{Methodology}

With approval from the Research Subject Review Board, patients between the ages of 10 and 18 years who had undergone an appendectomy between January 1, 2007 and December 31, 2011 were identified by CPT codes (44950, 44960 and 44970) from the Department of Surgery billing records, and their medical records were reviewed. Demographic information including age, gender, presenting temperature (fever defined as greater than 38 degrees Celsius), white blood cell count (WBC), use of preoperative imaging studies, operative reports, and pathologic findings were recorded. This particular age group was selected for study due to the relative uniform ability to articulate their symptoms and the ease in interpreting their physical findings.

Patients were excluded if they 1) had incomplete charts; 2) had a significant past medical history, including malignancy or immunosuppressive co-morbid conditions such as diabetes and solid organ transplantation; 3) had been transferred from outside institutions with imaging studies already performed; 4) had incidental appendectomies; or 5) had elective appendectomies done for chronic abdominal pain without a clearly defined etiology.

The included patients were categorized as Early (presenting prior to 7/1/2009) or Late (presenting after 7/1/2009) within our study time frame. They were also categorized based on the initial imaging study obtained. Those who received a CT as the primary study are designated as CT-First; those who received US as the primary study are designated as US-First. A small percentage of patients did not receive any preoperative imaging study. Patient outcomes were categorized as acute appendicitis (AA), perforated appendicitis (PA) and nega- tive appendectomy (NA) based on the review of operative reports and pathology reports. In cases of discrepancy between operative findings and pathologic reports, their clinical courses were further reviewed. Those who had postoperative ileus greater than 48 hours or required additional antibiotic therapy at the time of discharge were classified as PA; those who were discharged within 48 hours were classified as AA. The rates of acute appendicitis (AA), perforated appendicitis (PA), and negative appendicitis (NA) were calculated by dividing the number of cases of interest by the total number of cases in the relevant category.

Patient demographics and clinical features for the Early and Late patient populations were compared using the unpaired t-test. Patient demographics and clinical features for the Early and Late patient populations were compared using the unpaired t-test and the chi-squared test. Associations between time period (Early vs Late), patient outcome (AA or PA vs NA), receiving an imaging study (no imaging vs imaging), and the choice of imaging study (CT-first vs US-first) were analyzed using the chi-squared test. A value of $\mathrm{p}<0.05$ was considered to be significant. NA was used as the primary end point of analysis since these are patients who would not have undergone operative interventions if a correct diagnosis had been made.

\section{Results}

Between the years 2007 and 2011, a total of 762 patients between the ages of 10 to 18 years underwent appendectomies, and 586 of them met our inclusion criteria. Patient demographic characteristics and their disease processes are not significantly different between the Early and Late groups (Table 1). During the Early period, 150 patients $(49.3 \%)$ had undergone CT scans as their primary imaging modality prior to their appendectomy whereas only 52 patients (18.4\%) were in the CT-First category during the Late period. Of all US-first patients

Table 1. Demographic characteristics, clinical attributes, and patient outcomes of early and late study populations.

\begin{tabular}{cccc}
\hline & $\begin{array}{c}\text { Early Period } \\
(\mathbf{n}=\mathbf{3 0 4})\end{array}$ & $\begin{array}{c}\text { Late Period } \\
(\mathbf{n}=\mathbf{2 8 2})\end{array}$ & p-value \\
\hline Age, Mean \pm SD & $13.60 \pm 2.49$ & $13.76 \pm 2.64$ & NS \\
Male, n (\%) & $184(60.53)$ & $159(56.38)$ & NS \\
WBC, Mean \pm SD & $14.02 \pm 5.10$ & $13.63 \pm 4.72$ & NS \\
Fever, n (\%) & $6(1.97)$ & $9(3.19)$ & NS \\
LOS, Mean \pm SD & $2.34 \pm 2.83$ & $2.43 \pm 2.87$ & NS \\
AA, n (\%) & $223(73.36)$ & $221(78.37)$ & NS \\
PA, n (\%) & $58(19.08)$ & $45(15.96)$ & NS \\
NA, n (\%) & $23(7.57)$ & $16(5.67)$ & NS \\
\hline
\end{tabular}


( 86 boys and 93 girls), 60 (17 boys and 43 girls) received a subsequent $\mathrm{CT}$ scan.

The percentage of CT-first and US-first patients and their final diagnoses according to year are illustrated in Figures 1 and 2. Boys and girls were analyzed separately because of their anatomical differences and different clinical concerns. Figure 1 illustrates the transition in our practice pattern, and Figure 2 shows that patient disease patterns are similar between the years.

The correlation between the primary imaging modality and the eventual diagnosis of the patients are illustrated in Table 2. No significant difference in NA rates was found between the Early and Late periods for boys. Interestingly, girls had a significantly higher incidence of NA during the Early period (16 out of 120) compared to the Late period (6 out of 123). However, no statistically significant differences are observed in the NA rate when patients are segregated based on the decision to perform imaging (no imaging performed vs. imaging performed) or based on the imaging modality choice (CT-first vs. US-first).

We have successfully demonstrated that a change in

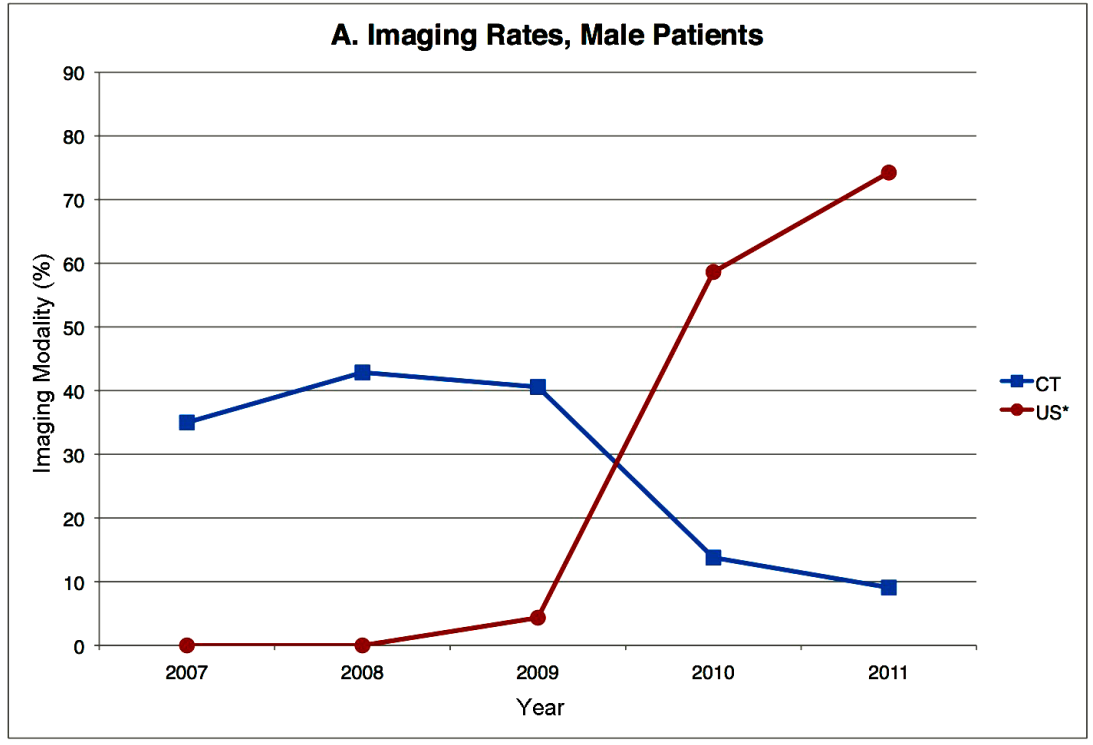

(a)

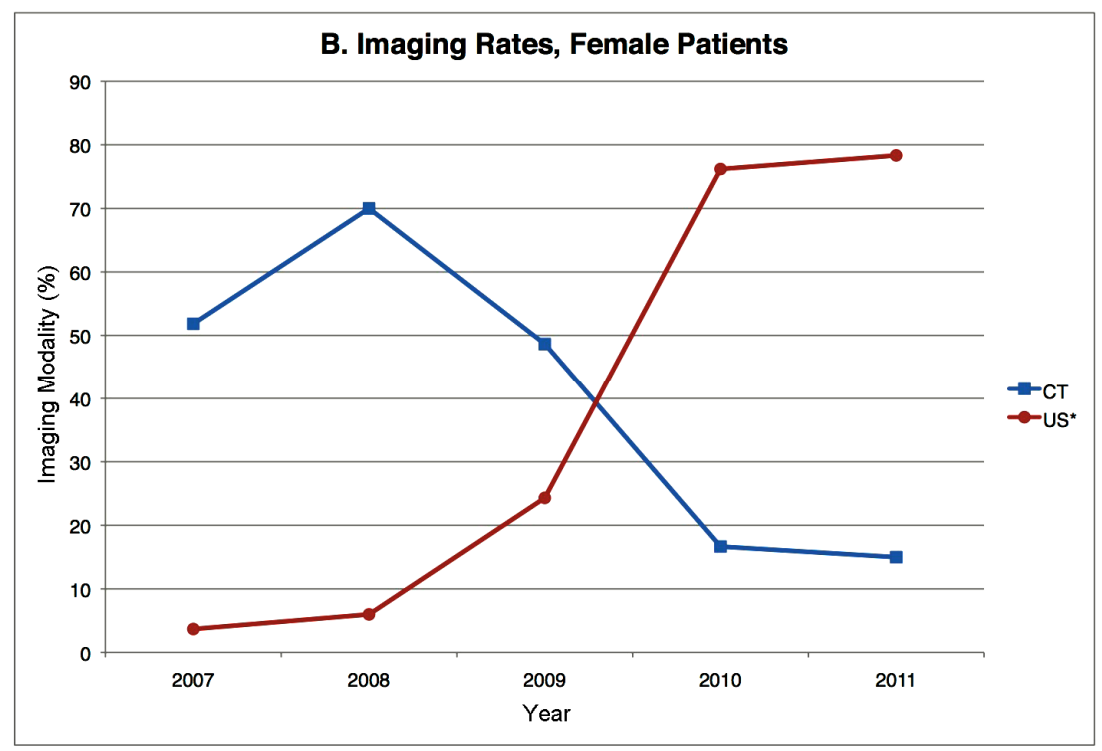

(b)

Figure 1. Yearly computed tomography and ultrasound imaging usage for boys and girls. The data shown represents the percentage of patients per year who received either a CT scan or an ultrasound as the initial preoperative imaging study. ${ }^{*}$ A subset of patients who initially received an ultrasound also received a follow-up CT scan. 


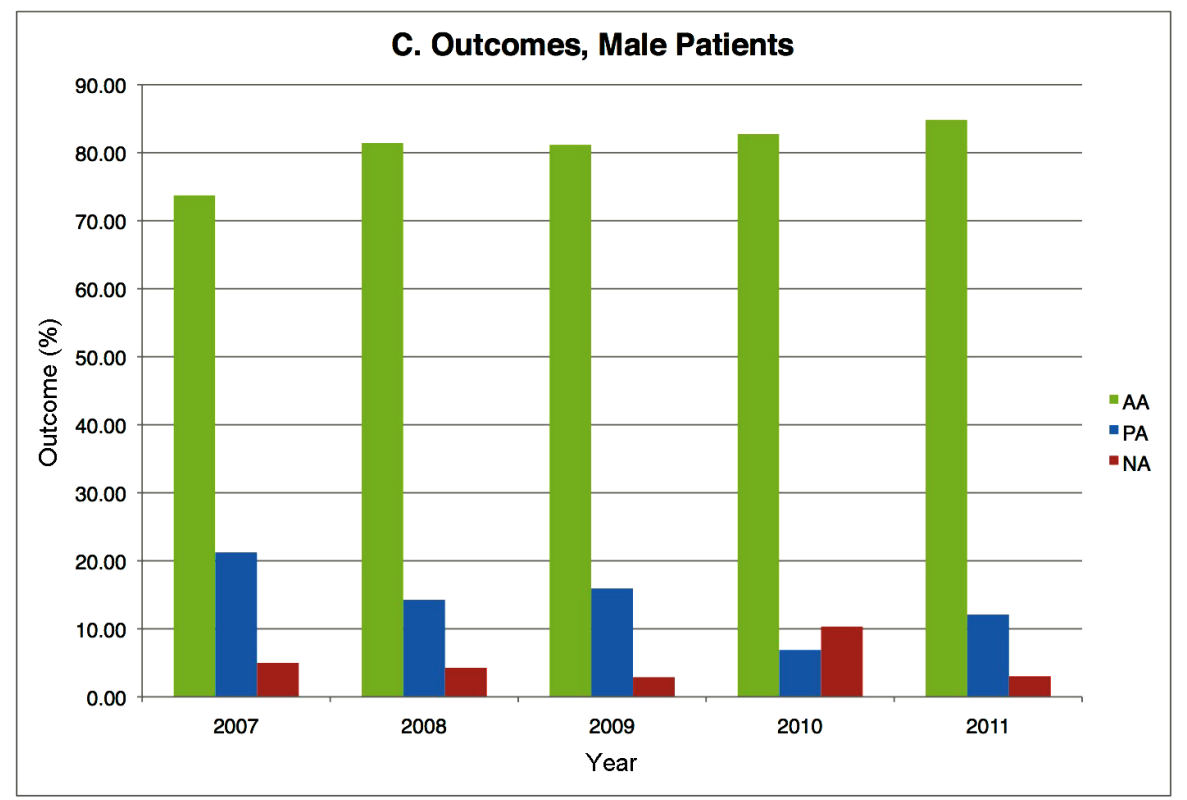

(a)

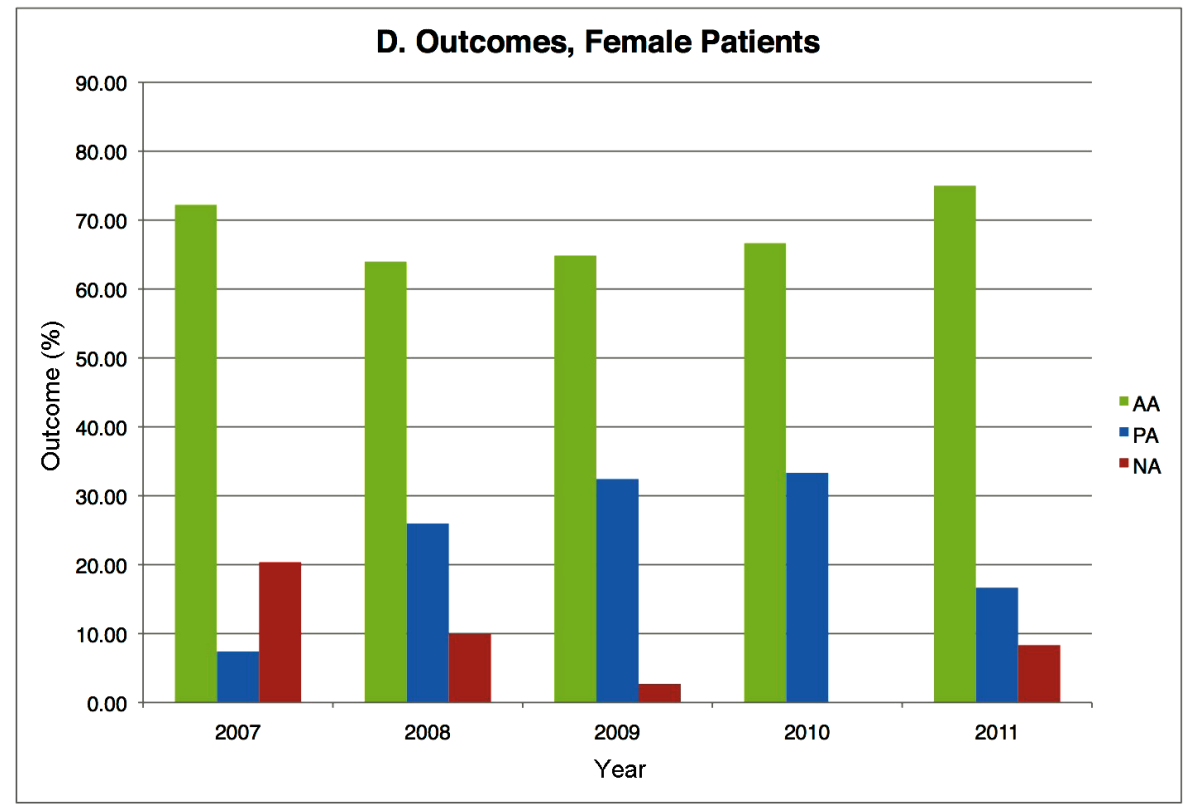

(b)

Figure 2. Yearly patient outcomes for boys and girls. The data shown represents the relative rates of acute appendicitis, perforated appendicitis, and negative appendicitis patients per year.

practice pattern in diagnostic work-up for appendicitis has not resulted in a change in outcome. Therefore, we conclude that the same level of diagnostic accuracy can be achieved with less radiation risk to our patients.

\section{Discussion}

This study confirms that an accurate history and a detailed physical exam remains the cornerstone for the di- agnosis of appendicitis in children. In fact, some authors have argued that imaging studies may delay surgical treatment and increase healthcare costs with no appreciable improvement in diagnostic accuracy or clinical outcomes over a simple evaluation by a skilled surgeon $[18,19]$. There is clearly an overreliance on using imaging studies to confirm this diagnosis in today's practice environment. Motivated by newly available information regarding the risks of radiation exposure from CT scan, 
Table 2. Comparisons of negative appendectomy rates between study groups.

\begin{tabular}{ccccc}
\hline & AA/PA & NA & p-value \\
\hline Boys & Early & 177 & 7 & NS \\
& Late & 149 & 10 & \\
& $\begin{array}{c}\text { No Imaging } \\
\text { Performed }\end{array}$ & 149 & 8 & NS \\
& $\begin{array}{c}\text { Imaging } \\
\text { Performed }\end{array}$ & 177 & 9 & \\
& CT-First & 97 & 3 & NS \\
Girls & US-First & 80 & 6 & \\
& Early & 104 & 16 & 0.026 \\
& $\begin{array}{c}\text { Late } \\
\text { No Imaging } \\
\text { Performed } \\
\text { Imaging } \\
\text { Performed } \\
\text { CT-First }\end{array}$ & 117 & 6 & \\
& 46 & 7 & NS \\
\hline
\end{tabular}

we have changed our practice pattern. Our study confirms that the indiscriminate use of CT imaging does not improve patient outcome. We are not able to explain the decrease in NA rates among girls during the Late period except surgeon's threshold for performing appendectomies.

The change in practice pattern was achieved via interpersonal and inter-departmental communications between the staff from the Departments of Surgery, Radiology and Emergency Medicine. All patients described herein had been evaluated at the Pediatric Emergency Department, and attending surgeons from both Divisions of Trauma and Acute Surgery and Pediatric Surgery performed appendectomies. There was no strict protocol or mandate that defined the criteria for choosing the imaging modality or any imaging at all. Hence, we are not able to offer further analysis regarding predictors for those who need CT scans after the initial US. For these patients, it would be more efficient to have obtained CT scans in the first place.

The major limitation of this study is that it does not address the population of patients for whom appendicitis was ruled out using radiographic imaging. This study also does not assess the efficiency of health resource utilization. Important factors for the management of pediatric appendicitis patients that have not been addressed in this study include: time from presentation to the definitive treatment, manpower requirement to perform either CT or US and the length of Emergency Department stay. The proper usage of radiographic imaging studies for acute appendicitis patients cannot be defined until there is a prospective study on differential diagnosis of acute pediatric abdominal pain with emphasis on radiographic imaging and appendicitis.

\section{REFERENCES}

[1] C. J. DeFrances and M. J. Hall, "2005 National Hospital Discharge Survey,” Advanced Data, No. 385, 2007, pp. 1-19.

[2] F. R. Lewis, J. W. Holcroft, J. Boey and E. Dunphy, “Appendicitis. A Critical Review of Diagnosis and Treatment in 1000 Cases," Archives of Surgery, Vol. 110, No. 5, 1975, pp. 677-684.

doi:10.1001/archsurg.1975.01360110223039

[3] R. G. Bachur, K. Hennelly, M. J. Callahan and M. C. Monuteaux, "Advanced Radiologic Imaging for Pediatric Appendicitis, 2005-2009: Trends and Outcomes,” Journal of Pediatrics, Vol. 160, No. 6, 2012, pp. 1034-1038. doi:10.1016/j.jpeds.2011.11.037

[4] M. K. Wolverson, B. Jagannadharao, M. Sundaram, P. F. Joyce, M. A. Riaz and J. B. Shields, "CT as a Primary Diagnostic Method in Evaluating Intraabdominal Abscess," American Journal of Roentgenology, Vol. 133, No. 6, 1979, pp. 1089-1095. doi:10.2214/ajr.133.6.1089

[5] S. L. Meshkov, S. E. Seltzer and H. J. Finberg, “CT Detection of Intraabdominal Disease in Patients with Lower Extremity Signs and Symptoms," Journal of Computer Assisted Tomography, Vol. 6, No. 3, 1982, pp. 497-501. doi:10.1097/00004728-198206000-00010

[6] M. A. Feldberg, M. J. Hendriks and P. F. van Waes, "Computed Tomography in Complicated Acute Appendicitis,” Gastrointestinal Radiology, Vol. 10, No. 3, 1985 , pp. 289-295. doi:10.1007/BF01893114

[7] P. L. Wagner, S. R. Eachempati, K. Soe, F. M. Pieracci, J. Shou and P. S. Barie, "Defining the Current Negative Appendectomy Rate: For Whom Is Preoperative Computed Tomography Making an Impact?” Surgery, Vol. 144, No. 2, 2008, pp. 276-282. doi:10.1016/j.surg.2008.03.040

[8] A. Deutsch and G. R. Leopold, "Ultrasonic Demonstration of the Inflamed Appendix: Case Report,” Radiology, Vol. 140, No. 1, 1981, pp. 163-164.

[9] S. G. Parulekar, "Ultrasonographic Findings in Diseases of the Appendix," Journal of Ultrasound in Medicine, Vol. 2, No. 2, 1983, pp. 59-64.

[10] J. B. Puylaert, “Acute Appendicitis: US Evaluation Using Graded Compression,” Radiology, Vol. 158, No. 2, 1986, pp. 355-360.

[11] B. A. Birnbaum and S. R. Wilson, "Appendicitis at the Millennium,” Radiology, Vol. 215, No. 2, 2000, pp. 337348.

[12] A. S. Doria, R. Moineddin, C. J. Kellenberger, M. Epelman, J. Beyene, S. Schuh and P. T. Dick, "US or CT for Diagnosis of Appendicitis in Children and Adults?” A Meta-Analysis. Radiology, Vol. 241, No. 1, 2006, pp. 8394. doi:10.1148/radiol.2411050913

[13] B. M. Pena and G. A. Taylor, “Radiologists' Confidence in Interpretation of Sonography and CT in Suspected Pe- 
diatric Appendicitis," American Journal of Roentgenology, Vol. 175, No. 1, 2000, pp. 71-74.

doi:10.2214/ajr.175.1.1750071

[14] D. Brenner, C. Elliston, E. Hall and W. Berdon, "Estimated Risks of Radiation-Induced Fatal Cancer from Pediatric CT," American Journal of Roentgenology, Vol. 176, No. 2, 2001, pp. 289-296. doi:10.2214/ajr.176.2.1760289

[15] D. J. Brenner, C. D. Elliston, E. J. Hall and W. E. Berdon, "Estimates of the Cancer Risks from Pediatric CT Radiation Are Not Merely Theoretical: Comment on Point/ Counterpoint: In X-Ray Computed Tomography, Technique Factors Should Be Selected Appropriate to Patient Size. Against the Proposition,” Medical Physics, Vol. 28, No. 11, 2001, pp. 2387-2388. doi:10.1118/1.1415074

[16] R. Krishnamoorthi, N. Ramarajan, N. E. Wang, B. Newman, E. Rubesova, C. M. Mueller and R. A. Barth, "Effectiveness of a Staged US and CT Protocol for the Diagnosis of Pediatric Appendicitis: Reducing Radiation Exposure in the Age of ALARA,” Radiology, Vol. 259,
No. 1, 2011, pp. 231-239. doi:10.1148/radiol.10100984

[17] N. Ramarajan, R. Krishnamoorthi, R. Barth, P. Ghanouni, C. Mueller, B. Dannenburg and N. E. Wang, "An Interdisciplinary Initiative to Reduce Radiation Exposure: Evaluation of Appendicitis in a Pediatric Emergency Department with Clinical Assessment Supported by a Staged Ultrasound and Computed Tomography Pathway," Academic Emergency Medicine, Vol. 16, No. 11, 2009, pp. 1258-1265. doi:10.1111/j.1553-2712.2009.00511.x

[18] A. E. Martin, D. Vollman, B. Adler and D. A. Caniano, "CT Scans May Not Reduce the Negative Appendectomy Rate in Children,” Journal of Pediatric Surgery, Vol. 39, No. 6, 2004, pp. 886-890. doi:10.1016/j.jpedsurg.2004.02.034

[19] D. York, A. Smith, J. D. Phillips and D. von Allmen, "The Influence of Advanced Radiographic Imaging on the Treatment of Pediatric Appendicitis,” Journal of Pediatric Surgery, Vol. 40, No. 12, 2005, pp. 1908-1911. doi:10.1016/j.jpedsurg.2005.08.004 